Article

\title{
Significantly Improved COVID-19 Outcomes in Countries with Higher BCG Vaccination Coverage: A Multivariable Analysis
}

\author{
Danielle Klinger ${ }^{1}$, Ido Blass ${ }^{2}$, Nadav Rappoport ${ }^{3, *(1)}$ and Michal Linial ${ }^{1, *(1)}$ \\ 1 Department of Biological Chemistry, Institute of Life Sciences, The Hebrew University of Jerusalem, \\ Jerusalem 91904, Israel; danielle.klinger@mail.huji.ac.il \\ 2 The Rachel and Selim Benin School of Computer Science and Engineering, \\ The Hebrew University of Jerusalem, Jerusalem 91904, Israel; ido.blass@mail.huji.ac.il \\ 3 Department of Software and Information Systems Engineering, Faculty of Engineering Sciences, \\ Ben Gurion University of the Negev, Be'er Sheva 84105, Israel \\ * Correspondence: nadavrap@bgu.ac.il (N.R.); michall@cc.huji.ac.il (M.L.); \\ Tel.: +972-74-779-5156 (N.R.); +972-54-882-0035 (M.L.)
}

Received: 9 June 2020; Accepted: 7 July 2020; Published: 11 July 2020

\begin{abstract}
The COVID-19 pandemic that started in China has spread within 3 months to the entire globe. We tested the hypothesis that the vaccination against tuberculosis by Bacille Calmette-Guérin vaccine (BCG) correlates with a better outcome for COVID-19 patients. Our analysis covers 55 countries complying with predetermined thresholds on the population size and number of deaths per million (DPM). We found a strong negative correlation between the years of BCG administration and the DPM along with the progress of the pandemic, corroborated by permutation tests. The results from multivariable regression tests with 23 economic, demographic, health-related, and pandemic restriction-related quantitative properties, substantiate the dominant contribution of BCG years to the COVID-19 outcomes. The analysis of countries according to an age-group partition reveals that the strongest correlation is attributed to the coverage in BCG vaccination of the young population (0-24 years). Furthermore, a strong correlation and statistical significance are associated with the degree of BCG coverage for the most recent 15 years, but no association was observed in these years for other broadly used vaccination protocols for measles and rubella. We propose that BCG immunization coverage, especially among the most recently vaccinated population, contribute to attenuation of the spread and severity of the COVID-19 pandemic.
\end{abstract}

Keywords: epidemiology; SARS-CoV-2; multivariable regression; tuberculosis; demography; coronavirus; MMR vaccine

\section{Introduction}

COVID-19 has spread within 3 months to 213 countries across the globe. The country-specific reports that are compiled daily by the World Health Organization (WHO) and made publicly available, provide statistical information on the number of tests performed, the number of confirmed cases, deaths and the cumulative state of patients hospitalized in serious and critical conditions [1]. Along with the spread of the pandemic, most countries imposed a policy of social distancing and other regulation to mitigate COVID-19 [2,3]. Despite the intense effort, key epidemiological parameters are still missing [4-9]. With 400,000 reported deaths and a world average of 51 deaths per million (DPM, June 6th, 2020), the death toll remains the most reliable measure for monitoring the spread and progression of the disease across countries [10]. While some European countries such as Belgium and the UK the DPM is $>500$, other infected countries (e.g., Hungary, Norway) are closer to the world 
average. The large differences in COVID-19 outcomes, even among neighboring countries (e.g., Spain and Portugal), are not likely to solely reflect differences in the regulations imposed by each country at the initial phase of the pandemic [11,12].

In this study, we tested the possibility that the extent and spreading of COVID-19 cases are associated with the status of tuberculosis (TB) immunization across the world. The Bacille Calmette-Guérin vaccine (BCG) contains a live attenuated strain of Mycobacterium bovis, is widely used to eradicate $\mathrm{TB}$ and was among the most broadly used vaccinations in the 20th century in neonatal and young children $[13,14]$. Currently, the BCG vaccine is provided to the entire population in most countries with high TB incidence [15]. Over the last two decades, numerous countries changed their policy and restricted BCG immunization policy to non-native born migrants from high TB burden countries [16]. Notably, numerous epidemiological and immunological studies demonstrate that BCG vaccination results in reduced morbidity and mortality to subsequent infections, presumably by its effect on the immune response [17]. Specifically, we questioned whether BCG vaccination regimens in different countries are linked with different COVID-19 outcomes. Our analysis considered a broad range of variables covering demography, economy, medical status, health system strength, and dynamics of the lockdown policy. We found that the BCG admission coverage of the young population is inversely correlated with COVID-19 outcomes. The implication of these observations on national policy for immunization is discussed.

\section{Materials and Methods}

\subsection{Data Extraction}

Information regarding COVID-19 outcomes was extracted daily between 29 January and 21 May 2020 from the Worldometers website [18] using a crawler written in Python software version 3.7 (https://www.python.org/downloads/release/python-370) [19].

Demographic measures of countries were extracted from the Worldometers website on 17 April 2020 [20]. Information regarding the share of population $>65$ years and economic development indicators were extracted from the World Bank data [21]. Information on educational management and school closure, as a measure of the quarantine status of the country, was extracted from the UNESCO institute of statistics dataset [22]. Prevalence of chronic diseases (e.g., obesity, type 2 diabetes) and the death rate from cardiovascular disorders were extracted from Our World In Data (ourworldindata.org) website. Table S1 provides the source for this country-related information.

Information regarding past and present BCG administration practices in every country was extracted from the BCG world atlas [20]. Two vaccination status groups were considered: (i) countries that had either a current or past national mandatory vaccination policy (49 in total), (ii) countries that have only administered BCG vaccinations to specific groups at risk (6 in total). In the latter group, only a negligible fraction of the population is BCG vaccinated [20]. In addition, the estimates for BCG, measles and rubella vaccination coverage between years 1980 and 2018 were extracted from the annual WHO reports [1]. For additional resources used to establish the years of mandatory BCG administration see Table S2.

\subsection{Data Analysis and Statistical Tests}

Countries were normalized by accounting for their population size (per $1 \mathrm{M}, \mathrm{PM})$. The normalized COVID-19 outcomes that were considered are death (DPM), positively validated cases (cases per million (CPM)), hospitalization with serious and critical conditions (SPM) and recovered (RPM). Accounting for the varying stages of the pandemic in each country, we define a unified aligned key date of a country as the first date when DPM reached for the first time the DPM value of 0.5 or higher. The following analysis was conducted across changing dates following the key aligned date (at a range of 10-50 days). In binary or categorized tests, we applied the ranked Wilcoxon test. For the continuous data, we applied linear regression. The regression fit and the calculated statistical significance ( $p$-value) for the COVID-19 
outcomes are reported. We tested the correlations between outcomes and years of BCG administration using Pearson's correlation, and reported the analytic $p$-values as well as permutation tests' $p$-values. Correlation robustness test was performed using repeating sub-sampling 2000 times $90 \%$ of countries. We report the high fraction of tests where correlations were significant ( $p$-value $<0.05)$. A correlation between the BCG by age groups was determined by partitioning the population of each country into three groups: (i) 24 years and younger; (ii) 25 to 64 years; (iii) 65 years and older. From the age partition and the BCG coverage within each age group, a value that measures the percentage of the population weighted by the share of the age group with BCG is calculated. All analyses were conducted in R software version 3.5 .2 (https://cran.r-project.org/bin/windows/base/old/3.5.2/) [23].

Further details on the statistical approach and the data processing are available in Text S1.

\section{Results}

\subsection{BCG Administration Years are Negatively Correlated with COVID-19 Outcomes}

In order to increase the robustness of the analysis, countries were included in the selected cohort if their population size was $>3 \mathrm{M}$, and they met the criteria of $\geq 3$ deaths per $1 \mathrm{M}$ population on 17 April 2020. Altogether, there are 134 countries with population size $>3 \mathrm{M}$. Among them, 55 complied with both thresholds, covering $62.9 \%$ of the world population. A regional partition of these countries is shown in Figure 1. For detailed information on the countries included in the analyses, see Table S3.
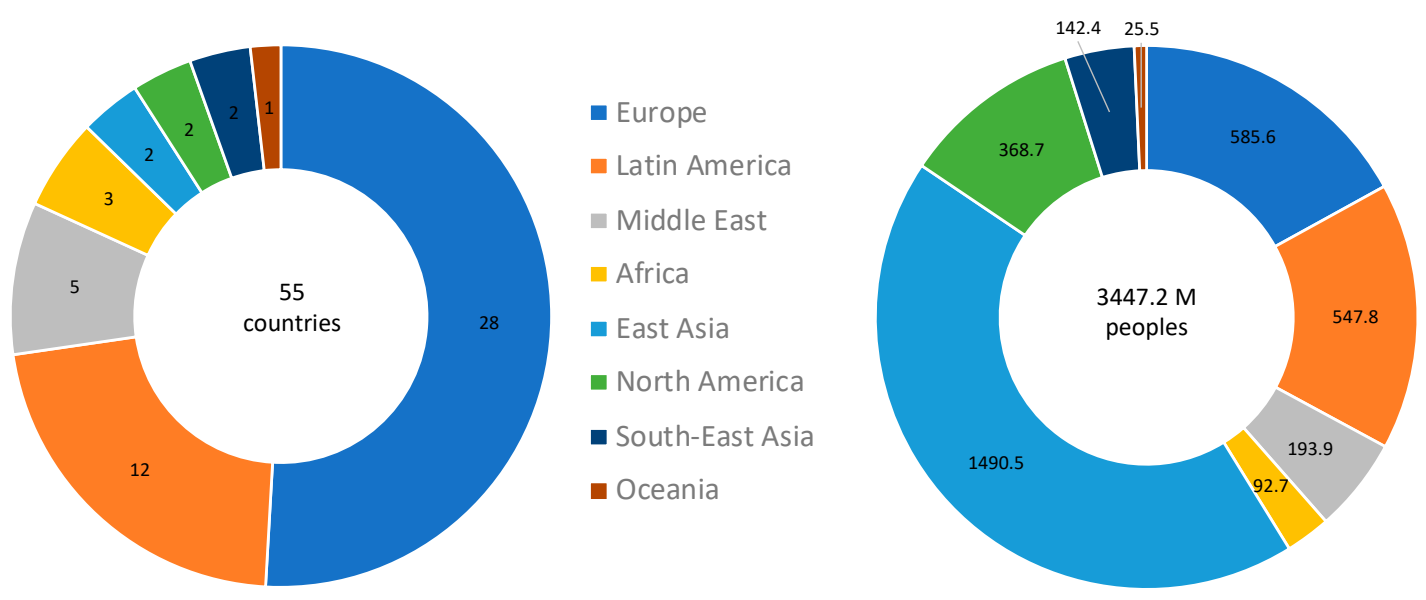

Figure 1. Countries analyzed in this study by geographical regions. Countries that comply with the predetermined thresholds for the population size and a minimum rate of deaths per million (DPM) at the analysis date are included. These countries ( 55 total) are partitioned by their geographical regions (left) and the cumulative population within each region (right).

First, we analyze COVID-19 outcomes as the difference in deaths or cases per million (DPM and CPM, respectively). Thus, the analysis was performed 20 days following two different alignments of key dates (defined by DPM $\geq 0.5$ and DPM $\geq 2$ ). Figure 2 shows strong and significant correlations between COVID-19 outcomes and the number of years of BCG administration. We observed a strong negative correlation with DPM outcome with $\mathrm{R}=-0.48$ ( $p$-value $=0.00056)$ and $-0.47(p$-value $=0.00084)$ when aligned at DPM threshold of 0.5 and 2, respectively (Figure 2a,b). Similarly, for the CPM as COVID-19 outcome, we observed a similar trend with $\mathrm{R}=-0.38(p$-value $=0.0091)$ and $-0.35(p$-value $=0.017)$ when aligned at DPM of $\geq 0.5$ and DPM $\geq 2$, respectively (Figure $2 c, d$ ). 


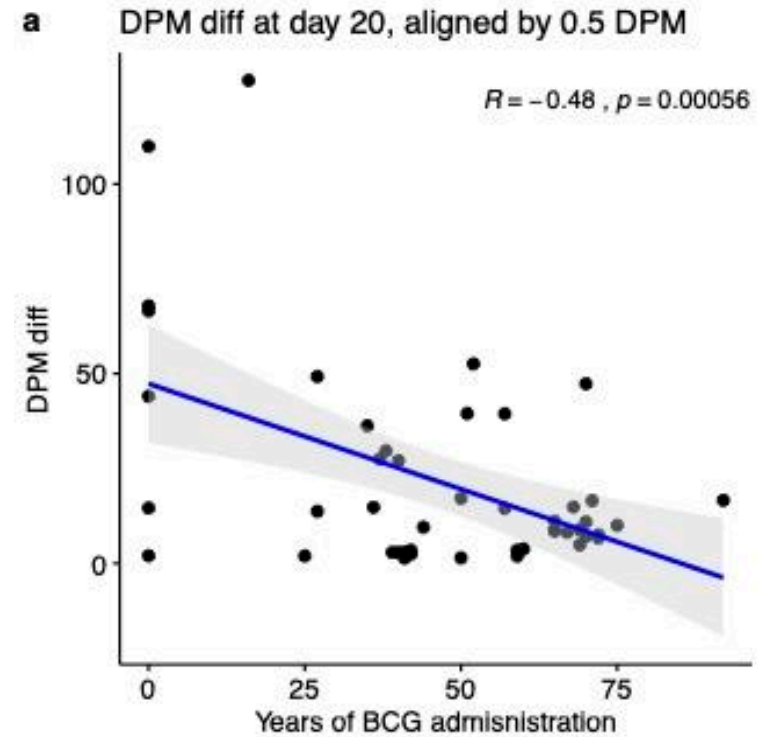

b DPM diff at day 20 , aligned by 2 DPM
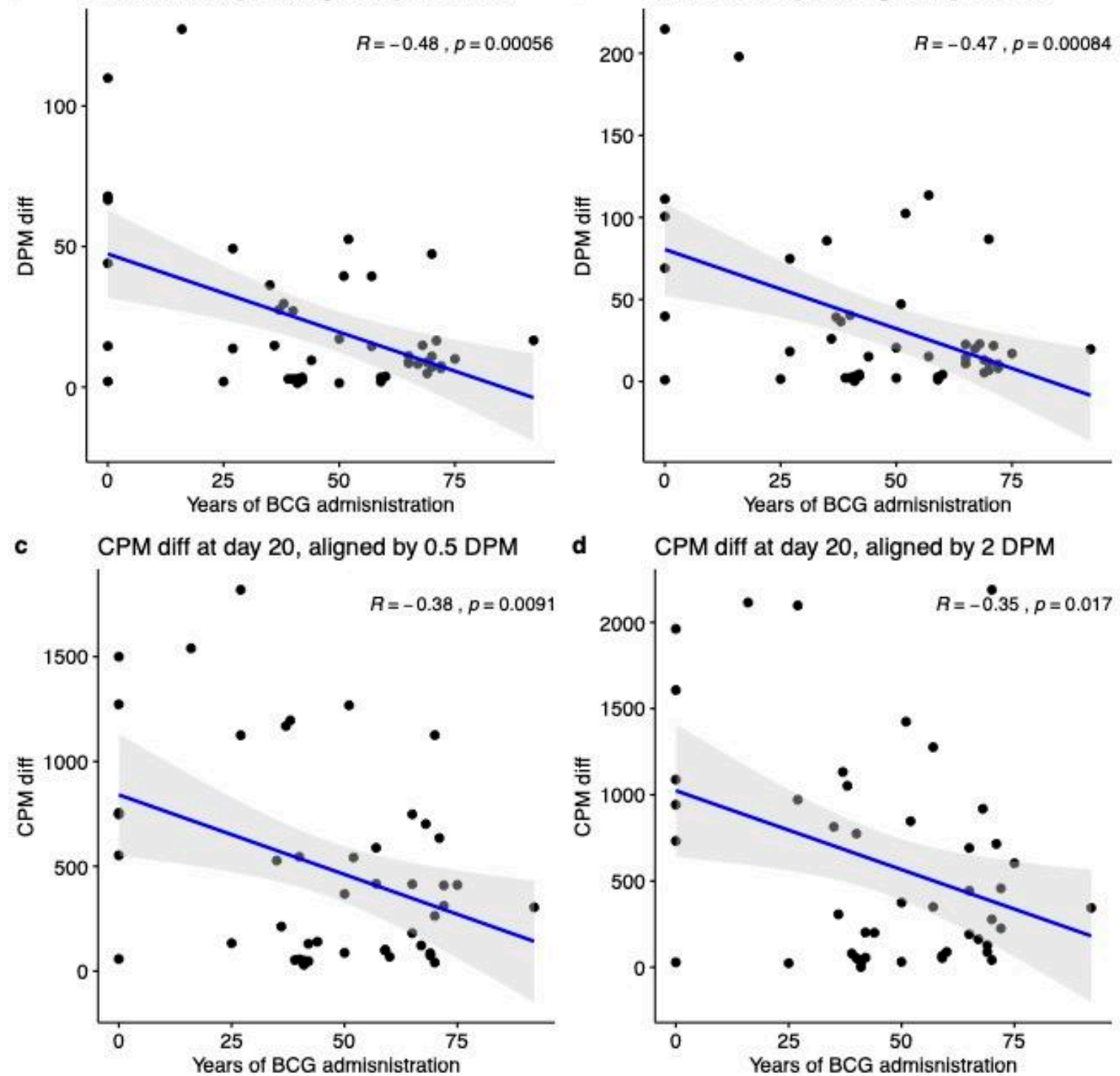

d CPM diff at day 20 , aligned by 2 DPM

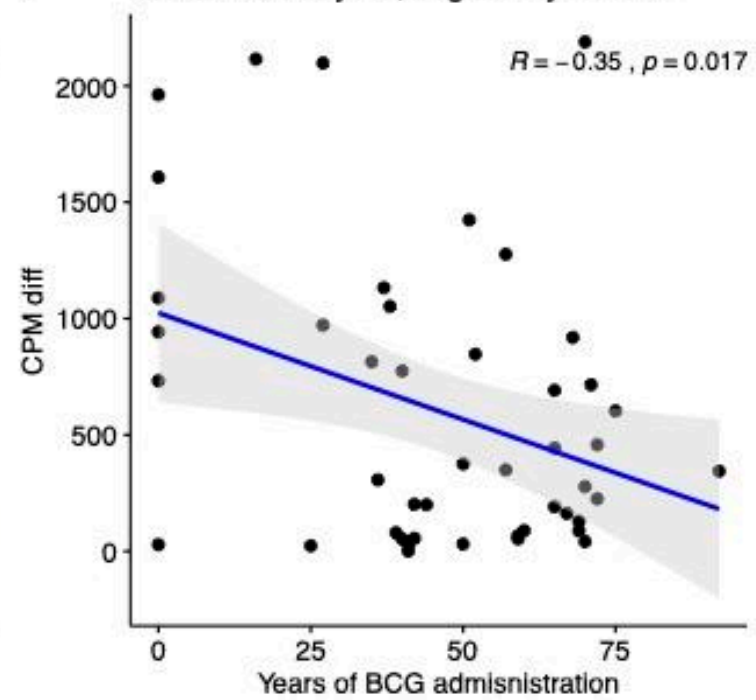

Figure 2. Statistical analysis of COVID-19 outcomes and years of Bacille Calmette-Guérin vaccine (BCG) administration. All correlations were measured at 20 days following the alignment key date. Correlations of years of BCG administration with DPM $=0.5$ (a) and DPM $=2(\mathbf{b})$. Correlation with cases per million (CPM) diff. at 20 days when the key date was defined as $C P M=0.5$ (c) and CPM $=2$ (d). DPM diff. and CPM diff. are calculated by the differences in the numbers from the measured date to alignment date. Shaded areas represent the $95 \%$ confidence intervals.

To test the generality of our observations we repeated the analysis at a broad range of time points along with the progress of the disease, starting from the 10th-day post alignment and showing the trends in 10-day intervals (10 to 50 days, Figure 3). For this analysis, we tested the outcomes of COVID-19 confirmed serious/critical cases (SPM) and the number of recovered (RPM), in addition to the DPM and CPM. The results of the DPM and SPM show a highly significant association for all time points, corroborated by the robust results obtained from performing 2000 permutation tests for each time interval for all tested outcomes (Table S4). An additional test for robustness was performed by repeating the 2000 correlation tests on $90 \%$ random subsamples of countries. We found that $4 / 5$ of the DPM- and SPM-examined dates presented significant results in more than $95 \%$ of random tests (Table S4). 


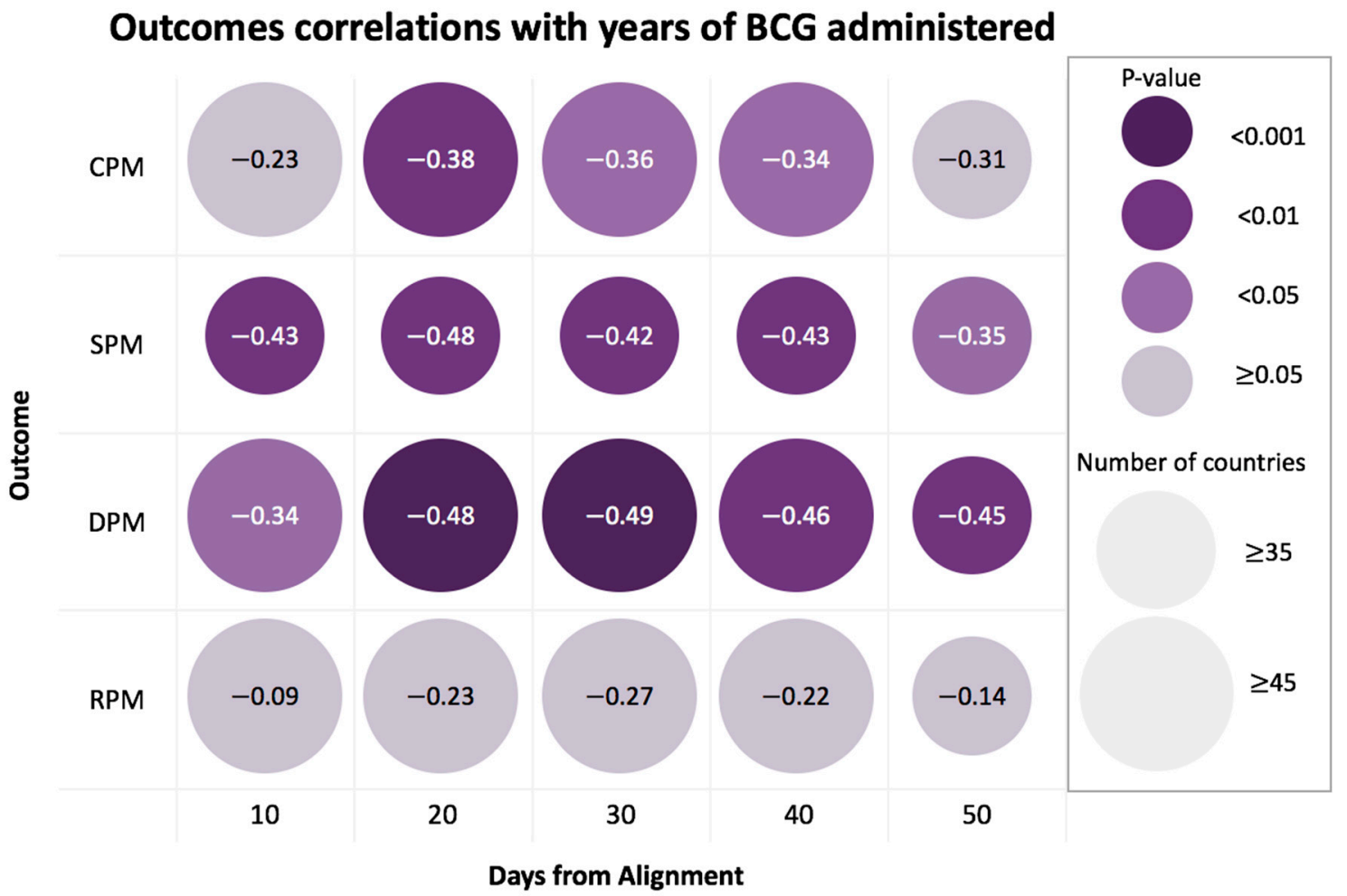

Figure 3. COVID-19 outcomes' correlations with years of BCG administration. Each row represents a different outcome. Each column represents the time interval of the outcome from the alignment date. Circle size depicts the number of countries, and color represents the statistical significance of the correlation with darker purple color indicating a higher significance. Values in circles are the correlation estimates where all the correlations have negative values. Note that while all countries reached 20 days post alignment of DPM $\geq 0.5$, at 50 days post alignment, some countries (5) that were still at an earlier phase of the pandemic at the alignment date failed to provide information. DPM, CPM, SPM and RPM stand for the number per million for death, validated cases, serious and critical conditions and recovered, respectively. For further details, see Table S4.

\subsection{Multivariable Analysis Reveals a Strong Contribution of BCG Administration to the COVID-19 Outcome Statistics}

Countries differ in many quantitative measurements like population size, Gross Domestic Product (GDP), lifespan, median age and more. To control for some of the potential confounding factors, we included numerous demographic values for a multivariable linear regression. The analysis included 23 demographic, economic, pandemic restriction-related and health-related country-based variables. The results show that the number of BCG administration years ranks consistently within the top two most significant coefficients and is within the top coefficients with the larger effect (as measured by the normalized beta coefficient, out of 23 coefficients) (Figure 4). The results are consistent among the different times observed (for further analysis see Table S5). Notably, a strong positive beta coefficient value is associated with the median age. This may be due to the fact that countries with a higher median age, a parameter reflective of the lifespan and the demographic bulges [2], have a higher fraction of older residents. Since a substantially higher risk of death is associated with infected older populations, countries with a higher median age are naturally susceptible to a higher percentage of deaths [24,25]. In accordance with COVID-19 age-associated risk, in the multivariable analysis the median age was associated with a strong positive coefficient. In addition, cancer percentage is also significant, and may reflect a confounding factor for lifespan and the rarity of cancer occurrence in the young population. The combined contribution of gender, chronic disease prevalence, and economy to the spread and fatality of COVID-19 was already reported [2], and will not be further discussed. 


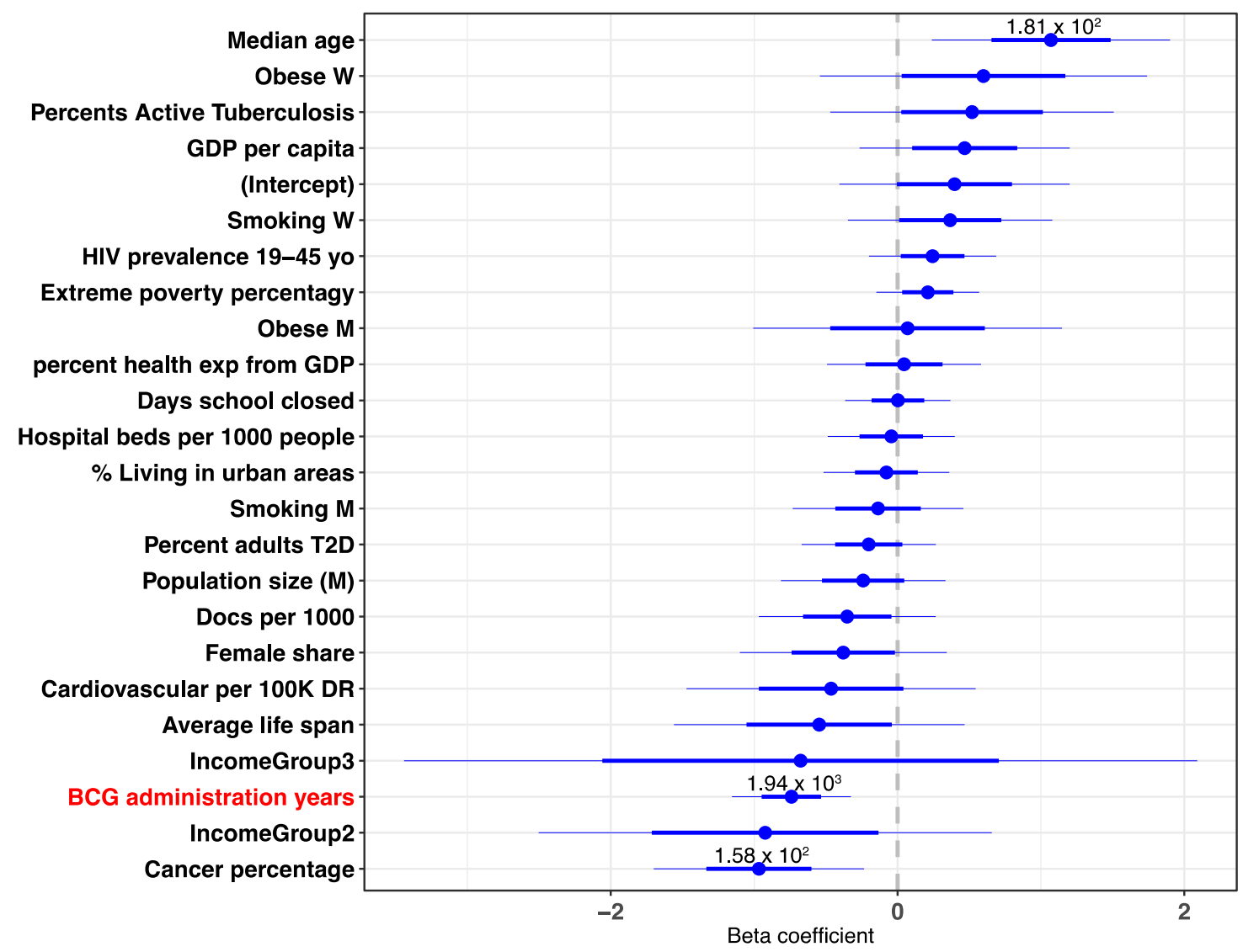

Figure 4. Multivariable analysis of country-centric quantitative data. Beta coefficients of the normalized multivariable linear regression for DPM at day 20 are shown. Blue lines represent the coefficients' $95 \%$ confidence intervals. $p$-values are shown for all variables with $p$-value $<0.1$.

\subsection{Highest Correlation with BCG Age Coverage Applies to the Most Recently Vaccinated}

Due to the varying effect of each age group on the viral spread in the population, we next investigated the relevance of age groups to the observation showing that years of BCG administration are strongly correlated with better COVID-19 outcomes. Epidemiological studies from COVID-19-positive cases in Shenzhen China confirm the importance of the young group age in the spread of the disease [26]. Specifically, children (1-16 years) were considered fundamental in the chain of transmission [27]. Figure 5 shows the correlation of total years of BCG administration with DPM difference according to the country-based age composition. The population in each country was partitioned to young ( $<24$ years of age), working-class (25-64 years) and old ( $>65$ years). The correlation with the young age group (tested at 20 days post-alignment key dates) shows the highest significance with $\mathrm{R}=-0.54$, $p$-value $=7.6 \times 10^{-5}$ (Figure 5a), and the correlation with the age group of 25-64 years (at 20 days) is also significant with $R=-0.32$, with a weaker significance ( $p$-value $=0.028$, Figure $5 b)$. Both correlations remain significant throughout a 50-day period post alignment. Remarkably, for the old age group ( $>65$ years), at all the time-frames tested, the correlation was negligible and insignificant (Figure $5 c$ ).

Notably, the age composition varies across countries. To examine the robustness of the results, we performed the same correlation analysis while not accounting for the actual fraction occupied by each of the age groups. The results ( 20 days post alignment date) are very similar to those obtained by weighting the fraction of the different age groups. Specifically, the correlation for the young age group is $\mathrm{R}=-0.58, p$-value $=2 \times 10^{-5}$; middle age group is $\mathrm{R}=-0.35, p$-value $=0.016$ and the old age group is $\mathrm{R}$ $=-0.14, p$-value $=0.34$. We further tested the statistical significance for the other outcomes (SPM, CPM and RPM), by age group according to population share, along different time points. Figure $5 \mathrm{~d}-\mathrm{f}$ show the dominant contribution of the young age group to the negative correlation at $10-50$ days 
post-alignment. Notably, the outcome of recovered per million (RPM) has a significant negative correlation only among the young group. The drop in RPM significance from 30 to 50 days is consistent with the epidemiological survey reporting on the long-time gap until recovery [28]. The middle age group (Figure $5 b, e$ ) is mostly insignificant and shows a borderline significance for the DPM and SPM as outcomes. All observations regarding the elderly (Figure $5 \mathrm{c}, \mathrm{f}$ ) are insignificant. We conclude that the elderly group does not contribute to the strong correlation with BCG administration.
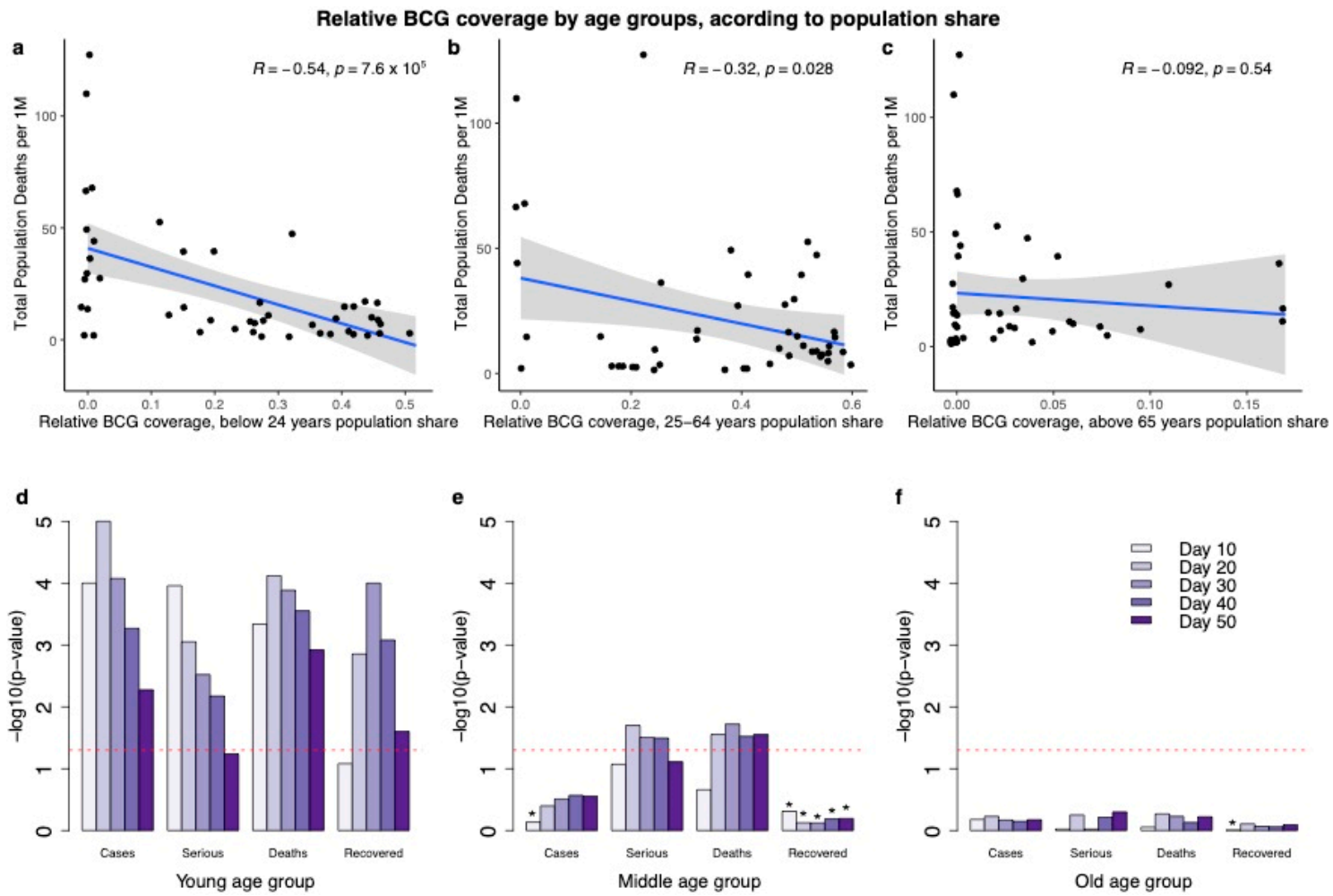

Figure 5. BCG coverage with respect to the DPM difference among three age groups. All correlations $(\mathbf{a}-\mathbf{c})$ and statistical significance $(\mathbf{d}-\mathbf{f})$ were measured following the DPM $\geq 0.5$ alignment key date. Relative BCG coverage is partitioned to three age groups, weighted by population share: (a) young (0-24 years), (b) middle age (25-64 years) and (c) old age group ( $>65$ years). Notice that data points in panels a-c were slightly moved horizontally to help distinguish overlapping symbols. The histogram $(\mathrm{d}-\mathrm{f})$ shows the statistical significance of the correlation of BCG years of administration for the 4 different COVID-19 outcomes according to the 3 age groups marked as: young (d) middle age (e) and elderly (f). Days from the key alignment date are colored from light to dark purple (10 to 50 days). The statistical significance is shown as $-\log 10$ ( $p$-value), the dashed red line indicates $p$-value of 0.05 . Asterisk represents the outcome with a positive correlation. All results with a positive correlation, marked by asterisks $\left({ }^{*}\right)$, are insignificant.

The pronounced signal in the young age group led us to investigate whether recent immunization may have a positive effect on the outcome. We divided the countries into three disjoined groups representing their vaccination policies over the past 15 years, disregarding the population share of the $0-15$ age group in each country: (i) countries with mandatory immunization policies over the past 15 years; (ii) countries with mandatory immunization policies, which were applied for less than 7.5 years within the past 15 years; (iii) countries with no mandatory immunization policies over the past 15 years (Figure 6a). Applying a test with DPM outcome, yielded highly significant results across all tested post-alignment (10-50 days) dates, establishing that countries with BCG immunization policies over the past 15 years have a significantly lower rate of DPM with respect with countries in group (iii). 

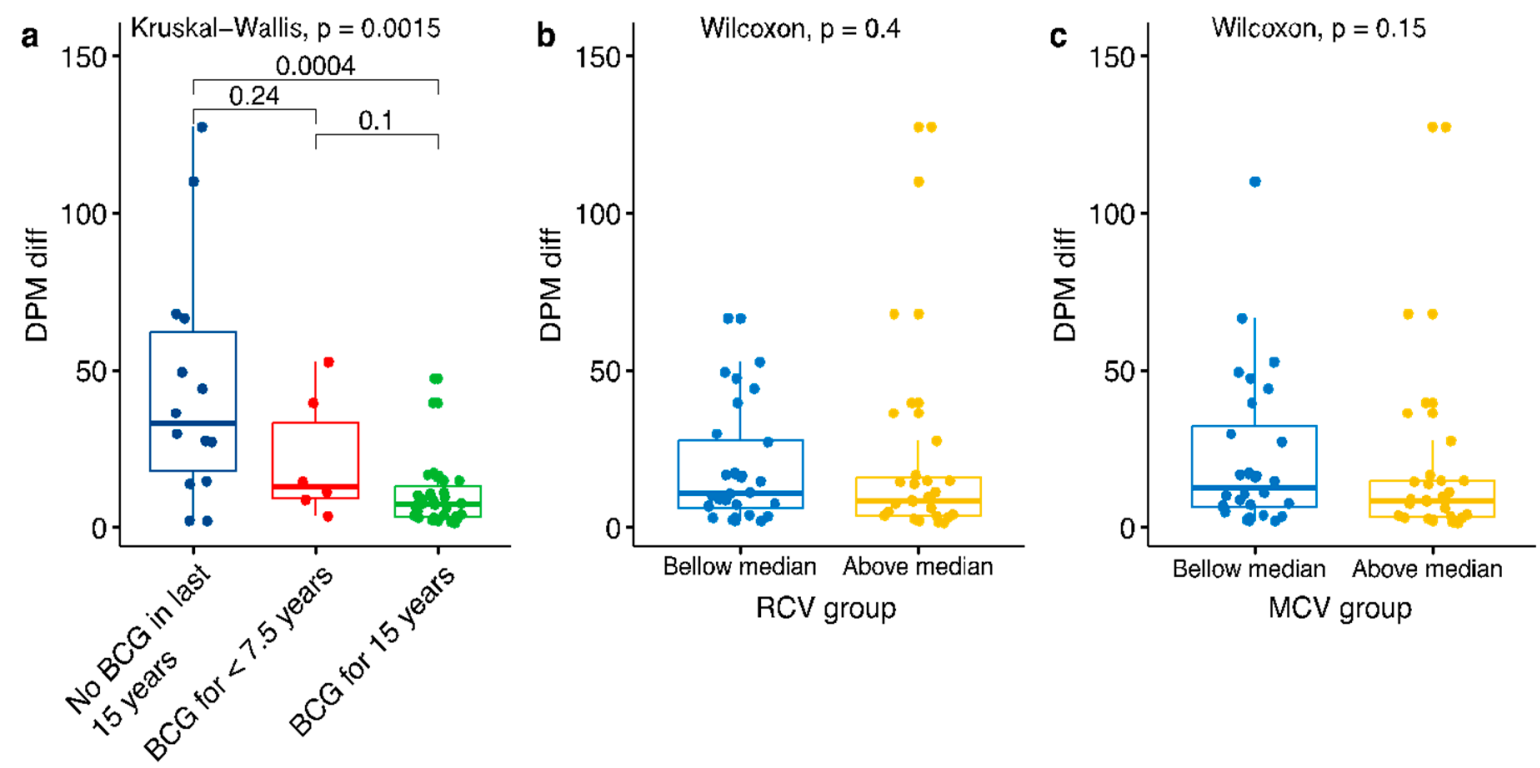

Figure 6. Immunization coverage for the last 15 years. (a) The DPM diff. (difference in DPM from the value at alignment key date) in countries that have (green), partially have (red), or have not (blue) rolled immunization BCG programs over the past 15 years. The statistical significance values are shown for each pair. (b) The statistics of RCV (rubella) and (c) MCV (measles) vaccines and COVID-19 DPM diff. according to the vaccination coverage. The partition of the countries is according to those above (yellow) and below (blue) median \% coverage for each of these tested vaccines. Data covering the past 15 years (2004-2018) was extracted from WHO reports. The statistical significance values are listed.

The significant result in the young age group raised the question of whether other immunizations might have a significant effect. To this end, we tested COVID-19 outcome and the globally used immunization against measles and rubella. We divided the countries into two groups representing their vaccination coverage over the past 15 years (as provided by the WHO): (i) countries with above median coverage; (ii) countries with below median coverage. While the world overall coverage of MCV1 (measles containing vaccine) is high in recent years, with an average of $88 \%$ in 2017, as provided by the WHO, the MCV1 coverage from 1990 shows substantial variation (e.g., $73.8 \%$ in India, 80.2\% in Italy, $85.7 \%$ in Algeria, $89.2 \%$ in Belgium) [1]. Applying the Wilcoxon test, yielded insignificant results at all tested dates (10-50 days post alignment). Opposite to the BCG results, we found no correlation between the degree of Measles and Rubella vaccination coverage and COVID-19 outcomes (Figure 6b,c).

\subsection{Data and Materials Availability}

All data needed to evaluate the conclusions in the paper are present in the paper and in the Supplemental Materials. An online tool for displaying the analytical results is available at: https://covi.shinyapps.io/COVID19/. It is a useful analytical webtool for single variant statistics, correlations, multivariable analyses and more. The user-friendly platform allows the changing of parameters by setting a threshold on population size, the time along the pandemic progression, selecting predetermined outcomes as a reference date for the alignment and changing the thresholds for alignment date. The code and data are available at: https://github.com/nadavrap/COVID19. Additional data and support related to this study may be requested from the authors.

\section{Discussion}

The significantly strong correlation between the BCG vaccination and better outcomes for COVID-19 is shown across many countries, covering the majority of the world population (Figure 1 and 
Table S1). The findings are based on an unbiased view of all countries that comply with predetermined thresholds for DPM and population size (see Materials and Methods).

The strong negative correlation between the BCG administration years and DPM was sustained at a range of time-points from the aligned-date (Figure 3). For testing the stability of the DPM correlation, we repeated the analysis for additional COVID-19 outcomes. We consider the number of hospitalized people (at a specific date) which were indicated by a serious or critical condition (SPM). This measurement is strongly dependent on the health care capacity and the actual phase of the pandemic. Using the SPM rather than the DPM as a measure shows that the trend of the BCG administration year remains stable and significant (Figure 3). As expected, the negative correlation to COVID-19 validated positive cases (CPM) is weaker relative to DPM. CPM is likely to reflect the capacity of different countries to carry out reliable molecular tests (PCR-based) or clinical tests (lung CT pathology) [29], and the national policy for targeted testing [30]. We observed no significant correlation for the country-level number of recovered (RPM). We attribute it to the non-standardized definition for COVID-19 recovery [25], the time delay for confirmed recovery [31]. Altogether, RPM is the least reliable outcome as the pandemic peak ranges greatly among countries.

Our multivariable analysis highlights the strength of combining a broad range of country-based quantitative observations. Among the analyzed variables are the economic measures [32], health system capacity (e.g., doctors per 1000 people), population composition, exposure to infectious diseases (e.g., prevalence of TB), pandemic restriction-related measures (i.e., school closure dates), major comorbidities (e.g., cancer, diabetes) and habits (e.g., smoking by sex). Many of these measures are correlated and may reflect confounding factors. Most importantly, the multivariable analysis validated the importance and statistical significance of BCG immunization years given all other variables (Figure 4). Policy toward quarantine, enforcement of isolation regulation (e.g., closure of cultural events, public transportation) were implemented at a country-based time point. For example, we included the number of days of closing the educational facilities relative to country alignment date as a variable in the analysis. It was used as a proxy for the level of the constraints imposed along the pandemic progression [33]. While it is expected to have a strong impact on COVID-19 spread $[25,34]$, this variable did not contribute to rejecting the hypothesis, and had a minimal impact on the multivariable analysis (Figure 4).

The exact date of BCG administration within each country, combined with the actual immunization coverage (provided by the WHO) and the population structure, allowed us to explicitly test the effect over time of the BCG immunization. Specifically, partitioning the population to young group, middle-age group and elderly confirmed that the strongest signal towards COVID-19 outcome is associated with young ( $<24$ years, Figure 5) and slightly also to the middle age group (25-64 years). However, the elderly ( $>65$ years) that are at the highest risk for COVID-19 mortality do not correlate with BCG higher coverage. The implication of our observation for COVID-19 epidemiology and pandemic dynamics is evident [25].

Our results suggest that in countries where the young population is vaccinated by BCG, a maximal protection is provided to the whole population. In countries where the young and middle-aged population groups were vaccinated, a significantly lower number of cases and deaths in the total population were observed (Figure 5). Universally, the DPM among young people is very low $(0.04 \%$ for $<17$ years) [18]. Therefore, the main contribution of the young age is with regards to the impact on the chain of infection $[27,35,36]$. Children and young adults (tested for ages 0-22 years) tend to make more social contacts than adults and hence are likely to contribute more to transmission than adults [35,37]. The middle-age group (25-64 years) overlaps with the group that is specified by an extensive cross-generation social interaction [37,38]. Thus, the higher BCG coverage of the young and middle-aged groups is associated with the attenuation of overall infection rate. The lack of correlation between BCG coverage for the elderly ( $>65$ years) and COVID-19 outcomes is in accord with the negligible impact of this population on viral transmission to the community $[27,35]$. 
Several reports proposed that BCG-vaccinated populations are resistant to viruses, and in particular toward SARS-CoV-2 [39]. Despite the broad usage of BCG for almost a century, and the underlying mode of action, the indirect long-term effect of BCG on the immune system remains enigmatic [40-42]. It is postulated that the positive effect of BCG immunization on COVID-19 outcomes is achieved by an improved systemic immunity which applies to the most recently vaccinated group. Accordingly, the younger age group that was recently vaccinated is likely to benefit from an immunological protection whereas the older age group (that was vaccinated by BCG over 65 years ago) are unlikely to display BCG-driven immune protection. We found no correlation between the degree of measles and rubella vaccination coverage and COVID-19 outcomes (Figure 6). These immunizations were shown to have some reduced susceptibility to viral infection [43].

There are several limitations that need to be addressed in supporting our main findings and the conclusions from this study. The first difficulty stems from the fact that countries vary greatly by their area, population density and age structure that can mask the apparent BCG protective effect [44]. Moreover, difference in culture and habits (e.g., religious gathering, social distancing, smoking), economy, demography and the capacity of the health system often cannot be easily generalized. In addition, our results may potentially be driven by a small number of influential countries.

To address some of these inherent difficulties, we duplicated the analyses for countries that were bounded by population size ( $>3 \mathrm{M}$ and $<100 \mathrm{M}$ ). We observed no effect on the main findings as shown in Figures S1 and S2. Moreover, an analysis that relies on COVID-19 static information is likely to suffer from unstable findings due to unpredicting trends on the pandemic dynamic [45,46]. By altering the threshold for the alignment date, we confirmed the robustness of the results (Figure 2). In addition, we tested for significance using sub-sampling tests. When randomly sampling $90 \%$ of the countries (with 2000 repeated tests), we found the 4/5 of the DPM- and SPM-examined dates presented significant results in more than $95 \%$ of random tests (Table S6).

The underlying mechanism by which BCG exerts its beneficial effect on infectious diseases is not fully resolved $[47,48]$. Recent evidence highlights the importance of reprograming of the innate system [49] and enhancing the response of heterologous Thelper cells [50]. Overall, the efficacy of BCG against TB is expected to cover approximately 15 years [51]. Thus, the strong statistical significance value for BCG being most effective for a recently immunized population argues that the active immunization phase rather than a residual non-specific protection from early-life events is associated with a better COVID-19 outcome [52,53].

Knowledge on the long-lasting effects of BCG on the immune system is essential for designing in-vivo experiments and ultimately effective vaccines. Some of the reported inconsistency with respect to the BCG-induced immune response was attributed to differences in the BCG strains, manufacturing methodology, and route of administration [54]. Several in-vitro assays showed that different sources of BCG are associated with a range of clinical efficacies [55]. Moreover, an improved protection for TB in rhesus macaques was reported for BCG that was admitted by repeated pulmonary mucosal delivery rather than by the default injection protocol [56]. We propose that future clinical trials for testing the impact of BCG on COVID-19 should consider the strain origin and the modes of BCG immunization.

Our results cannot exclude the possibility that a "pre-trained" state of immunity by BCG immunization exerts its positive effect, thus improving COVID-19 outcome at a population level. The finding that shows a strong and robust association of BCG coverage in the young age group with improved COVID-19 outcomes in the whole population calls for ongoing monitoring of the evolution of the pandemic world-wide. To this end, we developed a user-friendly platform with the capacity to change any of the dynamic parameters according to the pandemic progression.

\section{Conclusions}

We conclude that the inverse correlation with BCG administration years, the impact of a recent vaccination, and the validated role of the young population in the spread of COVID-19 calls for revisiting the global and national BCG immunization policy $[27,35,37]$. A growing number of clinical 
trials for testing the efficacy of BCG vaccination have been initiated. In one such trial, BCG was admitted once a month for 3 consecutive months in the elderly (60-75 years old) to test the prevention of acute upper respiratory tract infection. The results show significant prevention of infection in parallel to an improved response of T-helper cells [57]. Several on-going randomized controlled clinical trials cover different populations and several BCG strains. In one trial (named BADAS, USA), 1800 participates will be introduced to the BCG Tice strain, while in a larger trial (called BRACE, Australia) there will be over 10k healthcare workers tested by the Danish strain 1331. The goals in all these clinical trials are to determine if BCG vaccination reduces the incidence and severity of the COVID-19 pandemic [58,59]. While the WHO does not recommend BCG vaccination for prevention of COVID-19, we anticipate that the results of the BCG immunization clinical trials will provide guidelines for better controlling COVID-19 spread and severity. Without derogating from the importance of the results of clinical trials on the individual level, our opinion is that they will not necessarily directly affect country-wise epidemiological decisions. The reason is that minor results in clinical trials (such as 5\%) do not have the same effect at the individual level, in comparison with the population level-where even minor reductions in viral transmission can have major impact in terms of the pandemic's spread, population morbidity and mortality toll.

Supplementary Materials: The following are available online at http://www.mdpi.com/2076-393X/8/3/378/s1, Figure S1: correlation of BCG years of admission and COVID-19 outcomes for countries bounded by a population size of $>100 \mathrm{M}$, Figure S2: results from a multivariable analysis for countries bounded by a population size of $>100$ M, Table S1: variable resources across countries and populations, Table S2: additional resources on BCG administration years by country, Table S3: country cohort of 55 countries used in the study, Table S4: correlations and p-values of regression of years of BCG administrations and different outcomes including permutation tests, Table S5: multivariable results at numerous time points for BCG years of admission, Table S6: Sub-sampling of countries for years of BCG administration and COVID-19 outcome, Text S1: This includes supplementary methods and resources used in this study.

Author Contributions: D.K. coordinated the project. Conceptualization: D.K., N.R and M.L. Methodology: contributed equally by all co-authors. Data extraction: I.B. and D.K. Data design and software: I.B., D.K. and N.R. Interactive website: N.R. Code: I.B., D.H and N.R. Writing of the manuscript, reviewing and editing: D.K., N.R and M.L. Supervision, M.L. and N.R. All authors had full access to all of the data in the study and took responsibility for the integrity of the data and its accuracy. All authors have read and agreed to the published version of the manuscript.

Funding: This research received no external funding. The Center for Interdisciplinary Data Science (CIDR), at the Hebrew University partially contributed D.K and I.B fellowship.

Acknowledgments: We thank the biomedical community for valuable comments for the original version in MedRxiv. We thank Herve Bercovier for his comments and for sharing with us the fascinating history of the BCG. We thank Nati Linial and the Linial's lab for suggestions and fruitful discussions.

Conflicts of Interest: The authors declare no conflict of interest.

\section{References}

1. World Health Organization (WHO). Reported Estimates of BCG Coverage; World Health Organization: Geneva, Switzerland, 2019.

2. Dowd, J.B.; Andriano, L.; Brazel, D.M.; Rotondi, V.; Block, P.; Ding, X.; Liu, Y.; Mills, M.C. Demographic science aids in understanding the spread and fatality rates of COVID-19. Proc. Natl. Acad. Sci. USA 2020, 117, 9696-9698. [CrossRef]

3. Ebrahim, S.H.; Ahmed, Q.A.; Gozzer, E.; Schlagenhauf, P.; Memish, Z.A. Covid-19 and community mitigation strategies in a pandemic. BMJ 2020,368, m1066. [CrossRef]

4. Jung, S.-m.; Akhmetzhanov, A.R.; Hayashi, K.; Linton, N.M.; Yang, Y.; Yuan, B.; Kobayashi, T.; Kinoshita, R.; Nishiura, H. Real-time estimation of the risk of death from novel coronavirus (COVID-19) infection: Inference using exported cases. J. Clin. Med. 2020, 9, 523. [CrossRef]

5. Wang, Y.; Wang, Y.; Chen, Y.; Qin, Q. Unique epidemiological and clinical features of the emerging 2019 novel coronavirus pneumonia (COVID-19) implicate special control measures. J. Med. Virol. 2020, 92, 568-576. [CrossRef] 
6. Anderson, R.M.; Heesterbeek, H.; Klinkenberg, D.; Hollingsworth, T.D. How will country-based mitigation measures influence the course of the COVID-19 epidemic? Lancet 2020, 395, 931-934. [CrossRef]

7. Ruan, Q.; Yang, K.; Wang, W.; Jiang, L.; Song, J. Clinical predictors of mortality due to COVID-19 based on an analysis of data of 150 patients from Wuhan, China. Intensive Care Med. 2020, 46, 846-848. [CrossRef] [PubMed]

8. Bai, Y.; Yao, L.; Wei, T.; Tian, F.; Jin, D.Y.; Chen, L.; Wang, M. Presumed Asymptomatic Carrier Transmission of COVID-19. JAMA 2020, 323, 1406. [CrossRef] [PubMed]

9. Boldog, P.; Tekeli, T.; Vizi, Z.; Denes, A.; Bartha, F.A.; Rost, G. Risk Assessment of Novel Coronavirus COVID-19 Outbreaks Outside China. J. Clin. Med. 2020, 9, 571. [CrossRef] [PubMed]

10. Subbaraman, N. Why daily death tolls have become unusually important in understanding the coronavirus pandemic. Nature 2020. [CrossRef]

11. Onder, G.; Rezza, G.; Brusaferro, S. Case-fatality rate and characteristics of patients dying in relation to COVID-19 in Italy. JAMA 2020, 323, 1775-1776. [CrossRef]

12. Gilbert, M.; Pullano, G.; Pinotti, F.; Valdano, E.; Poletto, C.; Boelle, P.Y.; D'Ortenzio, E.; Yazdanpanah, Y.; Eholie, S.P.; Altmann, M.; et al. Preparedness and vulnerability of African countries against importations of COVID-19: A modelling study. Lancet 2020, 395, 871-877. [CrossRef]

13. Orme, I.M. Beyond BCG: The potential for a more effective TB vaccine. Mol. Med. Today 1999, 5, 487-492. [CrossRef]

14. Brewer, T.F.; Colditz, G.A. Relationship between bacille Calmette-Guerin (BCG) strains and the efficacy of BCG vaccine in the prevention of tuberculosis. Clin. Infect. Dis. 1995, 20, 126-135. [CrossRef] [PubMed]

15. Glaziou, P.; Sismanidis, C.; Floyd, K.; Raviglione, M. Global epidemiology of tuberculosis. Cold Spring Harb. Perspect. Med. 2015, 5, a017798. [CrossRef] [PubMed]

16. Pareek, M.; Greenaway, C.; Noori, T.; Munoz, J.; Zenner, D. The impact of migration on tuberculosis epidemiology and control in high-income countries: A review. BMC Med. 2016, 14, 48. [CrossRef]

17. Moorlag, S.; Arts, R.J.W.; van Crevel, R.; Netea, M.G. Non-specific effects of BCG vaccine on viral infections. Clin. Microbiol. Infect. 2019, 25, 1473-1478. [CrossRef]

18. WorldMeters. Published Online at Worldmeters.info, Dover. Available online: https://www.worldometers. info/coronavirus/ (accessed on 21 May 2020).

19. van Rossum, G. Python Tutorial, Technical Report CS-R9526, 1st ed.; Centrum Voor Wiskunde En Informatica (CWI): Amsterdam, The Netherlands, 1995.

20. Zwerling, A.; Behr, M.A.; Verma, A.; Brewer, T.F.; Menzies, D.; Pai, M. The BCG World Atlas: A database of global BCG vaccination policies and practices. PLoS Med. 2011, 8, e1001012. [CrossRef]

21. Bank, W. Population Ages 65 and Above (\% of Total Population). Available online: https://data.worldbank. org/indicator/SP.POP.65UP.TO.ZS/ (accessed on 18 April 2020).

22. Basilaia, G.; Kvavadze, D. Transition to online education in schools during a SARS-CoV-2 coronavirus (COVID-19) pandemic in Georgia. Pedagog. Res. 2020, 5, 1-9. [CrossRef]

23. R Core Team. R: A Language and Environment for Statistical Computing; R Foundation for Statistical Computing: Vienna, Austria, 2013.

24. Chen, T.; Wu, D.; Chen, H.; Yan, W.; Yang, D.; Chen, G.; Ma, K.; Xu, D.; Yu, H.; Wang, H.; et al. Clinical characteristics of 113 deceased patients with coronavirus disease 2019: Retrospective study. BMJ 2020, 368, m1091. [CrossRef]

25. Wu, J.T.; Leung, K.; Bushman, M.; Kishore, N.; Niehus, R.; de Salazar, P.M.; Cowling, B.J.; Lipsitch, M.; Leung, G.M. Estimating clinical severity of COVID-19 from the transmission dynamics in Wuhan, China. Nat. Med. 2020, 26, 506-510. [CrossRef]

26. Bi, Q.; Wu, Y.; Mei, S.; Ye, C.; Zou, X.; Zhang, Z.; Liu, X.; Wei, L.; Truelove, S.A.; Zhang, T. Epidemiology and transmission of COVID-19 in 391 cases and 1286 of their close contacts in Shenzhen, China: A retrospective cohort study. Lancet Infect. Dis. 2020. [CrossRef]

27. Kelvin, A.A.; Halperin, S. COVID-19 in children: The link in the transmission chain. Lancet Infect. Dis. 2020, 20, 633-634. [CrossRef]

28. Ma, S.; Zhang, J.; Zeng, M.; Yun, Q.; Guo, W.; Zheng, Y.; Zhao, S.; Wang, M.H.; Yang, Z. Epidemiological parameters of coronavirus disease 2019: A pooled analysis of publicly reported individual data of 1155 cases from seven countries. medRxiv 2020. [CrossRef] 
29. Ai, T.; Yang, Z.; Hou, H.; Zhan, C.; Chen, C.; Lv, W.; Tao, Q.; Sun, Z.; Xia, L. Correlation of chest CT and RT-PCR testing in coronavirus disease 2019 (COVID-19) in China: A report of 1014 cases. Radiology 2020. [CrossRef] [PubMed]

30. Cohen, J.; Kupferschmidt, K. Countries test tactics in 'war' against COVID-19. Am. Assoc. Adv. Sci. 2020, 367, 1287-1288. [CrossRef]

31. Wu, Z.; McGoogan, J.M. Characteristics of and important lessons from the coronavirus disease 2019 (COVID-19) outbreak in China: Summary of a report of 72314 cases from the Chinese Center for Disease Control and Prevention. JAMA 2020, 323, 1239-1242. [CrossRef]

32. Hopman, J.; Allegranzi, B.; Mehtar, S. Managing COVID-19 in low-and middle-income countries. JAMA 2020, 323, 1549-1550. [CrossRef]

33. Viner, R.M.; Russell, S.J.; Croker, H.; Packer, J.; Ward, J.; Stansfield, C.; Mytton, O.; Bonell, C.; Booy, R. School closure and management practices during coronavirus outbreaks including COVID-19: A rapid systematic review. Lancet Child Adolesc. Health 2020, 4, 397-404. [CrossRef]

34. Pan, A.; Liu, L.; Wang, C.; Guo, H.; Hao, X.; Wang, Q.; Huang, J.; He, N.; Yu, H.; Lin, X. Association of public health interventions with the epidemiology of the COVID-19 outbreak in Wuhan, China. JAMA 2020, 323, 1915. [CrossRef]

35. Davies, N.G.; Klepac, P.; Liu, Y.; Prem, K.; Jit, M.; group, C.C.-W.; Eggo, R.M. Age-dependent effects in the transmission and control of COVID-19 epidemics. Nat. Med. 2020. [CrossRef]

36. Qiu, H.; Wu, J.; Hong, L.; Luo, Y.; Song, Q.; Chen, D. Clinical and epidemiological features of 36 children with coronavirus disease 2019 (COVID-19) in Zhejiang, China: An observational cohort study. Lancet Infect. Dis. 2020, 20, 689-696. [CrossRef]

37. Liu, Y.; Gu, Z.; Xia, S.; Shi, B.; Zhou, X.N.; Shi, Y.; Liu, J. What are the underlying transmission patterns of COVID-19 outbreak? -An Age-specific Social Contact Characterization. EClinicalMedicine 2020, 22, 100354. [CrossRef] [PubMed]

38. Shim, E.; Tariq, A.; Choi, W.; Lee, Y.; Chowell, G. Transmission potential and severity of COVID-19 in South Korea. Int. J. Infect. Dis. 2020, 93, 339-344. [CrossRef] [PubMed]

39. Gursel, M.; Gursel, I. Is Global BCG vaccination coverage relevant to the progression of SARS-CoV-2 pandemic? Med. Hypotheses 2020, 109707. [CrossRef]

40. Bloom, B.R. BCG: Its Impact on Tuberculosis and Relevance to Autoimmune Disease. In The Value of BCG and TNF in Autoimmunity; Elsevier: Amsterdam, The Netherlands, 2018; pp. 1-10.

41. Butkeviciute, E.; Jones, C.E.; Smith, S.G. Heterologous effects of infant BCG vaccination: Potential mechanisms of immunity. Future Microbiol. 2018, 13, 1193-1208. [CrossRef]

42. Linehan, M.F.; Frank, T.L.; Hazell, M.L.; Francis, H.C.; Morris, J.A.; Baxter, D.N.; Niven, R.M. Is the prevalence of wheeze in children altered by neonatal BCG vaccination? J. Allergy Clin. Immunol. 2007, 119, 1079-1085. [CrossRef]

43. Salman, S.; Ahmed, M.S.; Ibrahim, A.M.; Mattar, O.M.; El-Shirbiny, H.; Sarsik, S.; Afifi, A.M.; Anis, R.M.; Agha, N.A.Y.; Abushouk, A.I. Intralesional immunotherapy for the treatment of warts: A network meta-analysis. J. Am. Acad. Dermatol. 2019, 80, 922-930. [CrossRef]

44. Bluhm, R.; Pinkovskiy, M. The spread of COVID-19 and the BCG vaccine: A natural experiment in reunified Germany. FRB N. Y. Staff Rep. 2020, 5, 926. [CrossRef]

45. Bodova, K.; Boza, V.; Brejova, B.; Kollar, R.; Mikusova, K.; Vinar, T. Time-adjusted analysis shows weak associations between BCG vaccination policy and COVID-19 disease progression. MedRxiv 2020. [CrossRef]

46. O'Neill, L.A.; Netea, M.G. BCG-induced trained immunity: Can it offer protection against COVID-19? Nat. Rev. Immunol. 2020, 20, 335-337. [CrossRef]

47. Netea, M.G.; van Crevel, R. BCG-induced protection: Effects on innate immune memory. Semin. Immunol. 2014, 26, 512-517. [CrossRef]

48. Dockrell, H.M.; Smith, S.G. What have we learnt about BCG vaccination in the last 20 years? Front. Immunol. 2017, 8, 1134. [CrossRef] [PubMed]

49. Kaufmann, E.; Sanz, J.; Dunn, J.L.; Khan, N.; Mendonca, L.E.; Pacis, A.; Tzelepis, F.; Pernet, E.; Dumaine, A.; Grenier, J.C.; et al. BCG educates hematopoietic stem cells to generate protective innate immunity against tuberculosis. Cell 2018, 172, 176-190. [CrossRef] 
50. Kleinnijenhuis, J.; Quintin, J.; Preijers, F.; Benn, C.S.; Joosten, L.A.; Jacobs, C.; van Loenhout, J.; Xavier, R.J.; Aaby, P.; van der Meer, J.W.; et al. Long-lasting effects of BCG vaccination on both heterologous Th1/Th17 responses and innate trained immunity. J. Innate Immun. 2014, 6, 152-158. [CrossRef] [PubMed]

51. Hart, P.D.; Sutherland, I. BCG and vole bacillus vaccines in the prevention of tuberculosis in adolescence and early adult life. Br. Med. J. 1977, 2, 293-295. [CrossRef]

52. Salman, S.; Salem, M.L. Routine childhood immunization may protect against COVID-19. Med. Hypotheses 2020, 140, 109689. [CrossRef] [PubMed]

53. Wang, F.; Nie, J.; Wang, H.; Zhao, Q.; Xiong, Y.; Deng, L.; Song, S.; Ma, Z.; Mo, P.; Zhang, Y. Characteristics of peripheral lymphocyte subset alteration in COVID-19 pneumonia. J. Infect. Dis. 2020, 221, 1762-1769. [CrossRef] [PubMed]

54. Tanner, R.; Villarreal-Ramos, B.; Vordermeier, H.M.; McShane, H. The humoral immune response to bcg vaccination. Front. Immunol. 2019, 10, 1317. [CrossRef]

55. Angelidou, A.; Diray-Arce, J.; Conti, M.G.; Smolen, K.K.; Van Haren, S.D.; Dowling, D.J.; Husson, R.N.; Levy, O. BCG as a case study for precision vaccine development: lessons from vaccine heterogeneity, trained immunity, and immune ontogeny. Front. Microbiol. 2020, 11, 332. [CrossRef] [PubMed]

56. Dijkman, K.; Sombroek, C.C.; Vervenne, R.A.W.; Hofman, S.O.; Boot, C.; Remarque, E.J.; Kocken, C.H.M.; Ottenhoff, T.H.M.; Kondova, I.; Khayum, M.A.; et al. Prevention of tuberculosis infection and disease by local BCG in repeatedly exposed rhesus macaques. Nat. Med. 2019, 25, 255-262. [CrossRef]

57. Wardhana, D.E.; Sultana, A.; Mandang, V.V.; Jim, E. The efficacy of Bacillus Calmette-Guerin vaccinations for the prevention of acute upper respiratory tract infection in the elderly. Acta. Med. Indones 2011, 43, 185-190. [PubMed]

58. de Vriese, J. Can a century-old TB vaccine steel the immune system against the new coronavirus. Science 2020. [CrossRef]

59. Ayoub, B.M. COVID-19 vaccination clinical trials should consider multiple doses of BCG. Die Pharm. 2020, 75, 159 .

(C) 2020 by the authors. Licensee MDPI, Basel, Switzerland. This article is an open access article distributed under the terms and conditions of the Creative Commons Attribution (CC BY) license (http://creativecommons.org/licenses/by/4.0/). 